\title{
The incidence of incision infections after lumbar fusion and the significance of dynamically monitoring serum albumin and C-reactive protein levels
}

\author{
Wenbin Jiang, Huagang Shi, Xuangeng Deng, Wei Hou, Dun Wan \\ Department of Spine Surgery, Sichuan Orthopaedic Hospital, Chengdu, China \\ Contributions: (I) Conception and design: W Jiang; (II) Administrative support: D Wan; (III) Provision of study materials or patients: H Shi; (IV) \\ Collection and assembly of data: W Jiang, X Deng, W Hou; (V) Data analysis and interpretation: W Jiang, H Shi; (VI) Manuscript writing: All \\ authors; (VII) Final approval of manuscript: All authors. \\ Correspondence to: Dun Wan. Department of Spine Surgery, Sichuan Orthopaedic Hospital, No. 132 West First Loop, Chengdu 610041, China. \\ Email: wandun2021@163.com.
}

Background To explore the incidence of incision infections and dynamically monitor albumin (ALB) and C-reactive protein (CRP) levels after lumbar fusion.

Methods: A total of 1,773 patients undergoing lumbar fusion in our hospital from March 2011 to March 2021 were selected and divided into the infection group and the non-infection group according to whether incision infections occurred postoperatively. The incidence of postoperative incision infections, the pathogenic characteristics and drug resistance of the pathogenic bacteria in patients in the infection group were analyzed. The general clinical data, serum ALB and CRP levels at different time points were compared between the two groups of patients, and the correlation between ALB and CRP levels was analyzed. ROC curve was used to evaluate the predictive value of serum ALB and CRP levels on the incidence of postoperative incision infections.

Results: Of the 1,773 patients, 41 (2.31\%) experienced postoperative incision infections. A total of 57 pathogens were detected, including 36 gram-negative bacteria (63.16\%), 18 gram-positive bacteria (31.58\%), and 3 fungi (5.26\%). Among gram-negative bacteria, Pseudomonas aeruginosa had the highest resistance to ampicillin. Among gram-positive bacteria, Stapbylococcus aureus had the highest resistance to penicillin and erythromycin. The age, BMI, the number of diabetes patients, levels of serum tumor necrosis factor-alpha (TNF- $\alpha$ ), interleukin-6 (IL-6) and procalcitonin (PCT) IL-8 at $1 \mathrm{~d}$ after operation in the infection group were significantly higher than those in the non-infection group $(\mathrm{P}<0.05)$. The serum ALB and CRP levels of the two groups differed over time, and the differences were statistically significant in terms of time, inter-group, time and inter-group interaction factors $(\mathrm{P}<0.05)$. Correlation analysis showed that ALB was significantly negatively correlated with CRP expression. Receiver operating characteristic (ROC) curve analysis showed that the area under the curve (AUC) value of combined ALB and CRP detection was 0.856, which was significantly higher than that of single detection with ALB or CRP $(\mathrm{P}<0.05)$.

Conclusions! Gram-negative bacterial infection was the main type of incision infection in patients after lumbar fusion. The changes in ALB and CRP levels were related to the incidence of postoperative incision infections.

Keywords: Lumbar fusion; wound infection; albumin (ALB); C-reactive protein (CRP); etiology

Submitted Aug 17, 2021. Accepted for publication Sep 28, 2021.

doi: 10.21037/apm-21-2512

View this article at: https://dx.doi.org/10.21037/apm-21-2512 


\section{Introduction}

Lumbar fusion is a commonly used surgical method for the treatment of lower back pain and spinal instability. It can significantly relieve the clinical symptoms of patients with lumbar degenerative diseases and reconstruct spinal stability $(1,2)$. Unfortunately, postoperative incision infection is a common complication of lumbar fusion, with an incidence of $0.7-20 \%$. This not only prolongs the treatment time, but also increases the risk of sepsis and even death, and thus, seriously affects the prognosis of patients (3). Therefore, early diagnosis and intervention of postoperative incision infections have important clinical significance. At present, the diagnosis of postoperative surgical incision infection is mainly based on the clinical symptoms, serological examination and incision secretion culture of patients, which is often lack of sensitivity and specificity, and there are shortcomings such as long inspection cycle, and there are limitations in the diagnosis of postoperative surgical incision infection (4). The studies on postoperative wound infection after lumbar fusion are mostly focused on the analysis of risk factors. It is believed that the increase of postoperative $\mathrm{C}$-reactive protein (CRP) level and the decrease of albumin (ALB) level are independent risk factors for postoperative wound infection $(5,6)$. However, there are few reports on the changes of the two and whether they can be used as predictor of postoperative wound infection. In order to improve the early diagnostic accuracy of surgical incision infection after lumbar fusion, this study analyzed incidence of surgical incision infections after lumbar fusion, the relationship between ALB and CRP levels and postoperative surgical incision infection, and evaluated its diagnostic value in the diagnosis of surgical incision infection after lumbar fusion. We present the following article in accordance with the STROBE reporting checklist (available at https://dx.doi.org/10.21037/apm-21-2512).

\section{Methods}

\section{General information}

A total of 1,773 patients who underwent lumbar fusion in our hospital from March 2011 to March 2021 were selected. Inclusion criteria were as follows: (I) clinical diagnosis of lumbar degenerative disease confirmed by imaging examination, with patients undergoing elective posterior lumbar fusion; (II) patients of any gender, aged >18 years old; (III) general clinical data were complete and available; and (IV) patients provided signed informed consent forms. Patients were excluded if they presented with the following: (I) malignant tumors, blood diseases or autoimmune diseases; (II) infectious lesions in the spine before the operation; (III) infections at other sites; and (IV) cognitive dysfunction rendering the patient unable to fully cooperate. All procedures performed in this study involving human participants were in accordance with the Declaration of Helsinki (as revised in 2013). The study was approved by ethics committee of Sichuan Orthopaedic Hospital (No. KY2020-043-01). Individual consent for this retrospective analysis was waived.

\section{Research metbods}

\section{General clinical data collection}

The general clinical characteristics of the participants including gender, age, body mass index (BMI), underlying diseases (diabetes, hypertension), smoking history, drinking history, operation method, operation time, intraoperative blood loss, postoperative drainage, postoperative indwelling time of urinary catheter and laboratory indicators were collated. The laboratory indexes included tumor necrosis factor- $\alpha$ (TNF- $\alpha$ ), interleukin-6 (IL-6) and procalcitonin (PCT) $1 \mathrm{~d}$ after operation.

\section{Evaluation of postoperative incision infection}

The presence of surgical incision infection within 30 days after the operation was diagnosed as follows: (I) fever of unknown origin (body temperature $\geq 38^{\circ} \mathrm{C}$ ), with chills, local redness, and swelling at the site of incision, and pain and itching with or without purulent material outflow; (II) positive bacterial culture from the surgical incision drainage fluid; (III) purulent secretion after the incision was naturally split or the surgeon opened the incision; and (IV) surgical exploration, histopathology, or imaging examination of deep incision abscess or other evidence of infection (7).

\section{Identification of bacterial strains and drug susceptibility test}

The wound secretions of the patients in the infection group were collected after debridement and submitted for bacterial culture within 1 hour after collection. The strains were identified by VITEK 2 Compact (BioMerieux, France) and drug sensitivity tests were performed using the Phoenix bacterial analyzer. The results of the (Kirby-Bauer) K-B disk method were used as supplementary experiments. The quality control strains were Staphylococcus aureus (ATCC25923), Pseudomonas aeruginosa (ATCC27853), and 
Table 1 Distribution of pathogens in patients with incision infections after lumbar fusion

\begin{tabular}{lcc}
\hline Group & Number of strains Proportion (\%) \\
\hline Gram-negative bacteria & 36 & 63.16 \\
Pseudomonas aeruginosa & 16 & 28.07 \\
Klebsiella pneumoniae & 7 & 12.28 \\
Escherichia coli & 6 & 10.53 \\
Acinetobacter baumannii & 4 & 7.02 \\
Enterobacter cloacae & 3 & 5.26 \\
Gram-positive bacteria & 18 & 31.58 \\
Staphylococcus aureus & 10 & 17.54 \\
Staphylococcus epidermidis & 6 & 10.53 \\
Others & 2 & 3.51 \\
Fungus & 3 & 5.26 \\
Total & 57 & 100.00 \\
\hline
\end{tabular}

Table 2 The resistance of gram-negative and gram-positive bacteria to antibiotics in patients with incision infections after lumbar fusion (n, \%)

\begin{tabular}{lcc}
\hline Antibacterial drug & $\begin{array}{c}\text { Pseudomonas } \\
\text { aeruginosa }(\mathrm{n}=16)\end{array}$ & $\begin{array}{c}\text { Staphylococcus } \\
\text { aureus }(\mathrm{n}=10)\end{array}$ \\
\hline Ampicillin & $14(87.50)$ & - \\
Cefepime & $10(62.50)$ & - \\
Ceftriaxone & $6(37.50)$ & - \\
Ciprofloxacin & $3(18.75)$ & $6(60.00)$ \\
Penicillin & - & $6(60.00)$ \\
Tetracycline & - & $5(50.00)$ \\
Azithromycin & - & \\
\hline
\end{tabular}

Escherichia coli (ATCC25922). All quality control strains were purchased from the Clinical Laboratory Center of the Health Commission.

\section{Detection of serum ALB and CRP levels}

To detect serum ALB and CRP levels, $5 \mathrm{~mL}$ fasting venous blood was collected pre-surgery and on postoperative days 1, 3, and 7. Samples were centrifuged at 3,500 rpm for 10 minutes, and the supernatant was collected and stored at low temperatures. An automatic biochemical analyzer (cobas8000, Roche, Germany) was used to detect serum ALB and CRP levels by immunoturbidimetry.

\section{Statistical analysis}

The SPSS 20.0 statistical software was used to analyze the data. The measurement data are expressed as mean \pm standard deviation $(\bar{x} \pm s)$, and independent sample $t$-tests were performed for comparison between the two groups. Variance analysis of repeated measurement data was performed for comparison at different time points within the group. The enumeration data are expressed as $\mathrm{n}(\%)$, and the $\chi^{2}$ test was performed. Pearson correlation analysis was used for correlation analysis. The application value of ALB and CRP in predicting postoperative incision infections was analyzed by receiver operating characteristic curve (ROC) with test level set to $\alpha=0.05$.

\section{Results}

\section{The incidence of postoperative incision infections and the} distribution of pathogens in patients with lumbar fusion

Among the 1,773 patients who received lumber fusion surgery, 41 experienced postoperative wound infections, and 1,732 did not. The incidence of postoperative wound infection was $2.31 \%$. Analysis of the 41 infected patients identified 57 strains of pathogenic bacteria, including 36 strains of gram-negative bacteria (63.16\%), 18 strains of gram-positive bacteria (31.58\%), and 3 strains of fungi (5.26\%). The distribution and proportion of pathogens are shown in Table 1.

\section{Analysis of drug resistance of the pathogenic bacteria in patients with incision infection after lumbar fusion}

Among the gram-negative bacteria, $P$. aeruginosa had the highest resistance to ampicillin. Among the grampositive bacteria, $S$. aureus had the highest resistance to penicillin and erythromycin, and no strains were resistant to vancomycin and linezolid (Table 2).

\section{A comparison of the clinical data between the infection group and the non-infection group}

The age, BMI, number of diabetic patients, and levels of serum tumor necrosis factor-alpha (TNF- $\alpha$ ), interleukin-6 (IL-6) and PCT IL-8 at $1 \mathrm{~d}$ after operation in the infection group were significantly higher than those in the noninfection group $(\mathrm{P}<0.05)$. There were no significant differences in gender, hypertension, smoking history, drinking history, operation method, operation time, 
Table 3 A comparison of the general clinical data between the two groups of patients [n (\%), $\bar{x} \pm \mathrm{s}$ ]

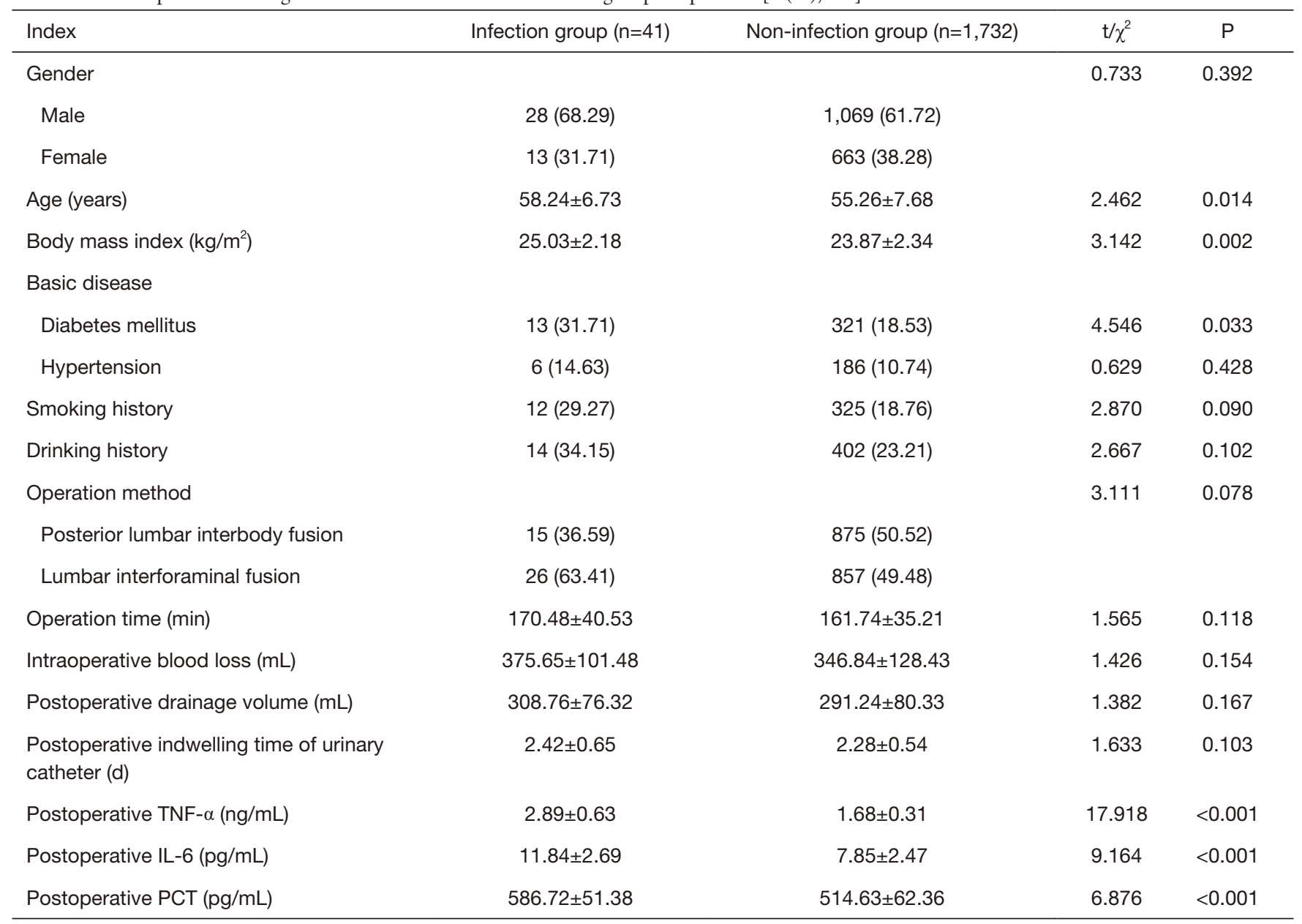

intraoperative blood loss, nor postoperative indwelling time between the two groups ( $\mathrm{P}>0.05$; Table 3).

\section{A comparison of serum ALB and CRP levels at different time points between the two groups}

The serum ALB and CRP levels of the two groups differed over time, and the differences were statistically significant in terms of time factors, inter-group factors, time and intergroup interaction factors $(\mathrm{P}<0.05)$. Before the operation, there were no significant differences in serum ALB and CRP levels between the two groups $(\mathrm{P}>0.05)$. At postoperative days 1,3 , and 7 , the serum ALB levels in the infection group were lower than that in the non-infection group, and the CRP levels were higher than that in the non-infection group $(\mathrm{P}<0.05$, Table 4$)$.

\section{Correlation analysis between serum ALB levels and CRP levels 3 days post-operation}

Correlation analysis revealed that ALB was significantly negatively correlated with CRP expression on postoperative day 3 ( $r=-0.476, \mathrm{P}=0.007$; Figure 1$)$.

\section{The predictive value of serum ALB and CRP on postoperative incision infection after lumbar fusion}

The ROC curve analysis showed that the area under the curve (AUC) values of ALB and CRP in predicting incision infections after lumbar fusion were 0.751 and 0.785 , respectively, with statistical significance $(\mathrm{P}<0.05)$. The sensitivity and specificity of combined ALB and CRP detection were $94.74 \%$ and $71.74 \%$, respectively, and the 
Table 4 A comparison of serum albumin and C-reactive protein levels at different time points between the two groups $(x \pm s)$

\begin{tabular}{|c|c|c|c|c|}
\hline Index & Infection group $(n=41)$ & Non-infection group $(n=1,732)$ & $\mathrm{t}$ & $\mathrm{P}$ \\
\hline Pre-operation & $35.87 \pm 4.23$ & $36.03 \pm 5.17$ & 0.197 & 0.844 \\
\hline Postoperative $1 \mathrm{~d}$ & $32.35 \pm 5.63$ & $34.15 \pm 4.38$ & 0.582 & 0.010 \\
\hline Postoperative $3 \mathrm{~d}$ & $29.15 \pm 3.71$ & $33.85 \pm 3.66$ & 8.120 & $<0.001$ \\
\hline $\mathrm{F}$ & \multicolumn{2}{|c|}{$F_{T}=43.913, F_{B}=28.476, F_{l}=7.502$} & & \\
\hline$P$ & \multicolumn{2}{|c|}{$\mathrm{P}_{\mathrm{T}}<0.001, \mathrm{P}_{\mathrm{B}}<0.001, \mathrm{P}_{\mathrm{I}}<0.001$} & & \\
\hline \multicolumn{5}{|l|}{ CRP (mg/L) } \\
\hline Pre-operation & $38.74 \pm 10.63$ & $36.64 \pm 9.95$ & 1.343 & 0.179 \\
\hline Postoperative $7 \mathrm{~d}$ & $61.38 \pm 11.32$ & $45.75 \pm 9.67$ & 10.340 & $<0.001$ \\
\hline $\mathrm{F}$ & \multicolumn{2}{|c|}{$\mathrm{F}_{\mathrm{T}}=491.724, \mathrm{~F}_{\mathrm{B}}=163.185, \mathrm{~F}_{\mathrm{l}}=42.431$} & & \\
\hline $\mathrm{P}$ & \multicolumn{2}{|c|}{$\mathrm{P}_{\mathrm{T}}<0.001, \mathrm{P}_{\mathrm{B}}<0.001, \mathrm{P}_{\mathrm{I}}<0.001$} & & \\
\hline
\end{tabular}

T, time; B, between groups; I, interaction (group $\times$ time). ALB, albumin; CRP, C-reactive protein.

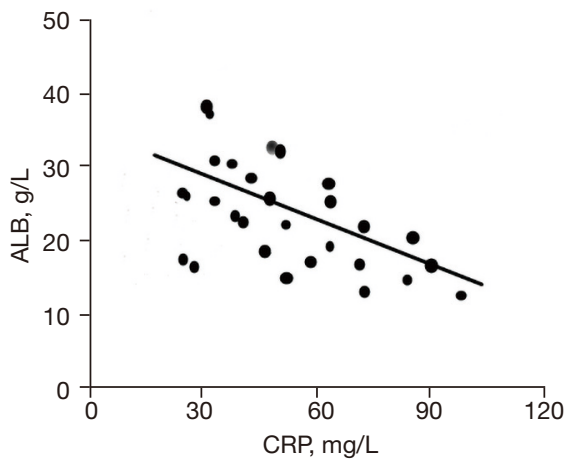

Figure 1 The correlation between serum ALB levels and CRP levels. ALB, albumin; CRP, C-reactive protein.

AUC value was 0.856 , which was significantly higher than that of ALB alone and CRP alone $(\mathrm{Z}=2.215,2.186, \mathrm{P}=0.027$, 0.034; Figure 2, Table 5).

\section{Discussion}

Lumbar fusion can fully relieve nerve root compression, restore spinal stability, and relieve associated pain. It is a common treatment for lumbar degenerative diseases such as lumbar spinal stenosis, lumbar disc herniation, and lumbar degenerative scoliosis (8). Unfortunately, postoperative

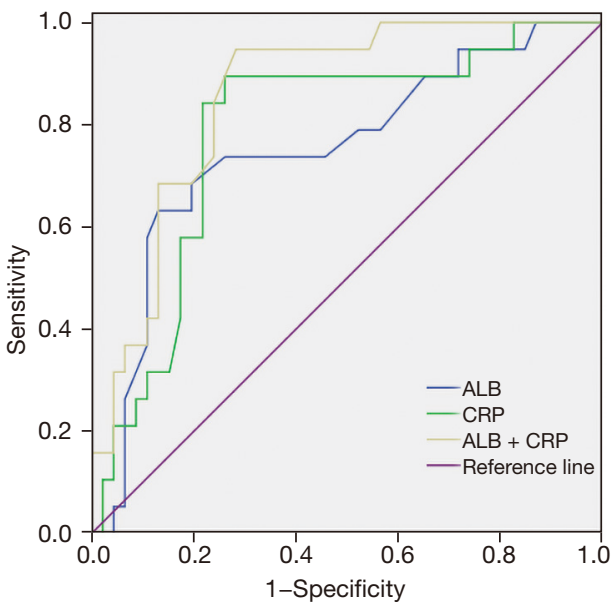

Figure 2 Receiver operating characteristic curve showing the predictive value of serum albumin and C-reactive protein levels at postoperative day 3 for predicting the incidence of incision infections after lumbar fusion. ALB, albumin; CRP, C-reactive protein.

incision infection is a common complication of lumbar fusion. A continuous cohort study on patients with posterior spinal internal fixation found that the incidence of postoperative incision infection was about $4.13 \%$ (9). Li et al. (10) demonstrated that the infection rate of lumbar 
Table 5 The predictive value of serum albumin and C-reactive protein on the incidence of postoperative incision infections after lumbar fusion

\begin{tabular}{lcccccc}
\hline Index & AUC & P & Cut-off value & Youden's index & Sensitivity (\%) & Specificity (\%) \\
\hline ALB & 0.751 & $<0.001$ & 30.10 & 0.501 & 73.16 & 76.96 \\
CRP & 0.785 & $<0.001$ & 100.50 & 0.634 & 89.47 & 73.91 \\
ALB + CRP & 0.856 & $<0.001$ & - & 0.665 & 94.74 & 71.74 \\
\hline
\end{tabular}

ALB, albumin; CRP, C-reactive protein; AUC, area under the curve.

fusion ranged from $1-20.0 \%$. In this study, the incidence of postoperative surgical incision infection was $2.31 \%$, infection rate lower than the above study. This may be related to the differences in the study cohorts including variations in preoperative risk factors and evaluation criteria. Studies have found that with the changes in environment and the wide application of antibiotics, the number of pathogens detected in postoperative incision infections are gradually increasing (11). In this current study, 57 strains of pathogenic bacteria were detected in 41 patients with infections, including 36 strains of gramnegative bacteria $(63.16 \%)$, with $P$. aeruginosa being the most common. There were 18 strains $(31.58 \%)$ of grampositive bacteria, with $S$. aureus being the most common. The results of drug sensitivity tests showed that $P$. aeruginosa had the highest resistance to ampicillin, and $S$. aureus had the highest resistance to penicillin and erythromycin. Thus, particular attention should be focused on these main types of pathogenic bacteria during clinical diagnosis. The drug resistance of pathogenic bacteria should be studied so that individualized treatment plans can be formulated.

This study revealed that the age, BMI, and the number of patients with diabetes, levels of serum TNF- $\alpha$, IL- 6 and PCT in the infection group were significantly higher than those in the non-infection group, suggesting that patients with postoperative incision infection had more serious inflammatory reactions, the age, obesity, and diabetes may increase the incidence of postoperative incision infections in patients with lumbar fusion. This may be related to the following reasons: (I) with an increase in age, the physical function and immune function of the patient gradually decreases, thereby increasing their risk of postoperative surgical incision infections (12); (II) patients with obesity have a thick layer of subcutaneous fat, and the postoperative wound aseptic gauze may be soaked by oily fat solutions, thereby increasing the postoperative wound infection rate (13); and (III) diabetes can increase the risk of vascular injury, incision tissue ischemia and hypoxia, and further aggravate postoperative incision infections
$(14,15)$. After infection, the levels of various inflammatory factors, including IL- 6 and TNF- $\alpha$, will increase, resulting in inflammatory cascade reactions, and high levels of inflammatory factors can aggravate oxidative stress injury and accelerate the infection process of patients. Therefore, we should pay close attention to the elderly, obesity, diabetes and other high-risk groups of postoperative infection, pay attention to the monitoring of postoperative inflammatory factors in patients, and adjust the treatment and nursing strategies in time to reduce the incidence of postoperative incision infection.

ALB is an important indicator for evaluating the nutritional status of patients, and it is also a key substance for maintaining blood colloid osmotic pressure (16). Studies have found that surgical stress can lead to loss of ALB or abnormal distribution, reducing the ability of patients to prevent pathogens (17). CRP is an acute phase protein synthesized by the liver. In the early stages of infection, it increases rapidly due to the activation of inflammatory factors, and accelerates organ damage. Both CRP and ALB can be used as evaluation indexes for the prognosis of infected patients $(18,19)$. However, there is a lack of monitoring of the dynamic changes of ALB and CRP in the early stage of patients after lumbar fusion in the current clinical practice, and there is application value in predicting the occurrence of postoperative incision infection remains to be further studied. The results of this study showed that the serum ALB and CRP levels of the two groups differed over time. At postoperative days 1, 3, and 7, the serum ALB levels of the infection group were lower than that of the control group, and the CRP levels were higher than that of the non-infection group. Correlation analysis showed that there was a significant negative correlation between ALB and CRP expression levels. Furthermore, serum ALB and CRP levels were significantly related to the incidence of surgical incision infections after lumbar fusion. Lower ALB level and higher CRP level will increase the risk of infection in patients. The ROC curve showed that the AUC value of combined ALB and CRP detection was 0.856, which was 
significantly higher than that of ALB alone or CRP alone as single index detection factors. This further indicated that ALB and CRP are related to the incidence of surgical incision infections after lumbar fusion. Indeed, combined detection of ALB and CRP may improve the early predictive efficiency of postoperative incision infections. Yamamoto et al. (20) also found that hypoproteinemia during hospitalization was associated with postoperative surgical site infection in patients with spinal surgery. Thus, when serum ALB levels decrease, intervention measures for correcting hypoproteinemia should be considered. This present study suggested that early diagnosis of surgical incision infection in patients after lumbar fusion may be facilitated by monitoring ALB and CRP levels in the clinic, and relevant prevention and control measures should be actively implemented to reduce the incidence of infections and improve the prognosis of patients.

In summary, patients with incision infections after lumbar fusion were mainly infected by gram-negative bacteria, and the incidence of infections was high. The changes in serum ALB and CRP levels were related to the incidence of postoperative incision infections, and ALB and CRP may be effective indicators for the early prediction of incision infections in patients after lumbar fusion.

\section{Acknowledgments}

Funding: None.

\section{Footnote}

Reporting Checklist: The authors have completed the STROBE reporting checklist. Available at https://dx.doi. org/10.21037/apm-21-2512

Data Sharing Statement: Available at https://dx.doi. org/10.21037/apm-21-2512

Conflicts of Interest: All authors have completed the ICMJE uniform disclosure form (available at https://dx.doi. org/10.21037/apm-21-2512). The authors have no conflicts of interest to declare.

Ethical Statement: The authors are accountable for all aspects of the work in ensuring that questions related to the accuracy or integrity of any part of the work are appropriately investigated and resolved. All procedures performed in this study involving human participants were in accordance with the Declaration of Helsinki (as revised in 2013). The study was approved by ethics committee of Sichuan Orthopaedic Hospital (No. KY2020-043-01). Individual consent for this retrospective analysis was waived.

Open Access Statement: This is an Open Access article distributed in accordance with the Creative Commons Attribution-NonCommercial-NoDerivs 4.0 International License (CC BY-NC-ND 4.0), which permits the noncommercial replication and distribution of the article with the strict proviso that no changes or edits are made and the original work is properly cited (including links to both the formal publication through the relevant DOI and the license). See: https://creativecommons.org/licenses/by-nc-nd/4.0/.

\section{References}

1. Ye I, Tang R, White SJ, et al. Predictors of 30-Day Postoperative Pulmonary Complications After Open Reduction and Internal Fixation of Vertebral Fractures. World Neurosurg 2019;123:e288-93.

2. Benson JC, Vizcaino MA, Kim DK, et al. Exophytic Lumbar Vertebral Body Mass in an Adult with Back Pain. AJNR Am J Neuroradiol 2020;41:1786-90.

3. Bible JE, Biswas D, Devin CJ. Postoperative infections of the spine. Am J Orthop (Belle Mead NJ) 2011;40:E264-71.

4. Brunsting JY, Pille FJ, Oosterlinck M, et al. Incidence and risk factors of surgical site infection and septic arthritis after elective arthroscopy in horses. Vet Surg 2018;47:52-9.

5. Lenski M, Tonn JC, Siller S. Interleukin-6 as inflammatory marker of surgical site infection following spinal surgery. Acta Neurochir (Wien) 2021;163:1583-92.

6. Yuwen $\mathrm{P}, \mathrm{Chen} \mathrm{W}, \mathrm{Lv} \mathrm{H}$, et al. Albumin and surgical site infection risk in orthopaedics: a meta-analysis. BMC Surg 2017;17:7.

7. Mangram AJ, Horan TC, Pearson ML, et al. Guideline for Prevention of Surgical Site Infection, 1999. Centers for Disease Control and Prevention (CDC) Hospital Infection Control Practices Advisory Committee. Am J Infect Control 1999;27:97-132; quiz 133-4; discussion 96.

8. Mohamad B, Alvi MA, Jake B, et al. Impact of Opioid Dependence on Economic Burden of Hospitalizations Among Patients Undergoing Lumbar Fusion: Insights from the National Inpatient Sample. Neurosurgery 2020;67: 447-9.

9. Inose H, Kobayashi $Y$, Yuasa M, et al. Procalcitonin and Neutrophil Lymphocyte Ratio After Spinal 
Instrumentation Surgery. Spine (Phila Pa 1976) 2019;44:E1356-61.

10. Li Z, Liu P, Zhang C, et al. Incidence, Prevalence, and Analysis of Risk Factors for Surgical Site Infection after Lumbar Fusion Surgery: $\geq 2$-year Follow-up Retrospective Study. World Neurosurg 2019;131:e460-e7.

11. Gustafsson K, Tatz AJ, Slavin RA, et al. Intraincisional medical grade honey decreases the prevalence of incisional infection in horses undergoing colic surgery: A prospective randomised controlled study. Equine Vet J 2021;53:1112-8.

12. Tyagi V, Kahan J, Huang P, et al. Negative Pressure Incisional Therapy and Postoperative Infection after Posterior Approach Primary Total Hip Arthroplasty. Cureus 2020;12:e7394.

13. Andrés-Cano P, Cerván A, Rodríguez-Solera M, et al. Surgical Infection after Posterolateral Lumbar Spine Arthrodesis: CT Analysis of Spinal Fusion. Orthop Surg 2018;10:89-97.

14. Wang J, Chen K, Li X, et al. Postoperative adverse events in patients with diabetes undergoing orthopedic and general surgery. Medicine (Baltimore) 2019;98:e15089.

15. Maruyama H, Kusachi S, Makino H, et al.

Postoperative Infection after Colorectal Surgery:

Subanalysis of Data from the 2015 Japan Postoperative

Cite this article as: Jiang W, Shi H, Deng X, Hou W, Wan D. The incidence of incision infections after lumbar fusion and the significance of dynamically monitoring serum albumin and C-reactive protein levels. Ann Palliat Med 2021;10(10):1087010877. doi: 10.21037/apm-21-2512
Infectious Complications Survey. J Nippon Med Sch 2020;87:204-10.

16. Sodavadiya KB, Singh S, Agrawal BK. Role of preoperative assessment of serum cholesterol and albumin in surgical site infection. Int J Med Biomed Studies 2019;3:112-8.

17. Zhang F, Liu X, Tan Z, et al. Effect of postoperative hypoalbuminemia and supplement of human serum albumin on the development of surgical site infection following spinal fusion surgery: a retrospective study. Eur Spine J 2020;29:1483-9.

18. Ogihara S, Yamazaki T, Shiibashi M, et al. Risk Factor Analysis of Deep Surgical Site Infection After Posterior Instrumented Fusion Surgery for Spinal Trauma: A Multicenter Observational Study. World Neurosurg 2020;134:e524-9.

19. Tsantes AG, Papadopoulos DV, Lytras T, et al. Association of malnutrition with surgical site infection following spinal surgery: systematic review and meta-analysis. J Hosp Infect 2020;104:111-9.

20. Yamamoto Y, Shigematsu H, Iwata E, et al. Hypoalbuminemia Increased the Length of Stay in the Treatment of Postoperative Acute Surgical Site Infection in Spinal Surgery. Spine (Phila Pa 1976) 2020;45:E1564-71.

(English Language Editor: J. Teoh) 